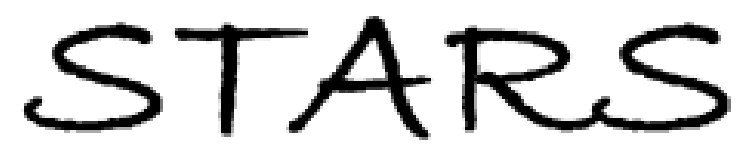

University of Central Florida

STARS

$1-1-2000$

\title{
Comparison of infrared frequency selective surfaces fabricated by direct-write electron-beam and bilayer nanoimprint lithographies
}

\author{
Irina Puscasu \\ University of Central Florida \\ G. Boreman \\ University of Central Florida \\ R. C. Tiberio \\ D. Spencer \\ R. R. Krchnavek
}

Find similar works at: https://stars.library.ucf.edu/facultybib2000

University of Central Florida Libraries http://library.ucf.edu

This Article; Proceedings Paper is brought to you for free and open access by the Faculty Bibliography at STARS. It has been accepted for inclusion in Faculty Bibliography 2000s by an authorized administrator of STARS. For more information, please contact STARS@ucf.edu.

\section{Recommended Citation}

Puscasu, Irina; Boreman, G.; Tiberio, R. C.; Spencer, D.; and Krchnavek, R. R., "Comparison of infrared frequency selective surfaces fabricated by direct-write electron-beam and bilayer nanoimprint lithographies" (2000). Faculty Bibliography 2000s. 2749.

https://stars.library.ucf.edu/facultybib2000/2749

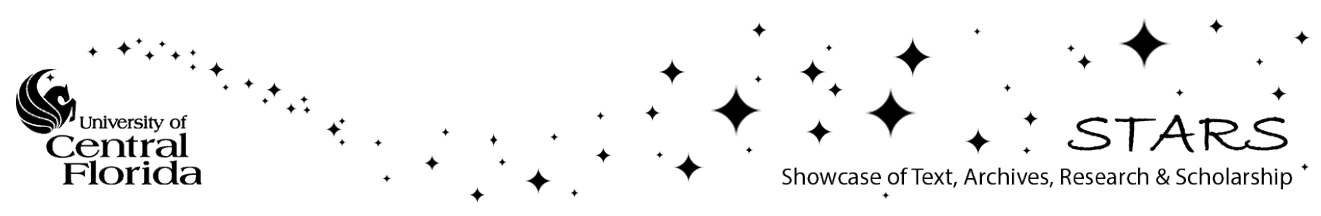




\section{Comparison of infrared frequency selective surfaces fabricated by direct-write electron-beam and bilayer nanoimprint lithographies}

Irina Puscasu, G. Boreman, R. C. Tiberio, D. Spencer, and R. R. Krchnavek

Citation: Journal of Vacuum Science \& Technology B: Microelectronics and Nanometer Structures Processing, Measurement, and Phenomena 18, 3578 (2000); doi: 10.1116/1.1319838

View online: https://doi.org/10.1116/1.1319838

View Table of Contents: https://avs.scitation.org/toc/jvn/18/6

Published by the American Institute of Physics

\section{ARTICLES YOU MAY BE INTERESTED IN}

Infrared frequency selective surfaces fabricated using optical lithography and phase-shift masks Journal of Vacuum Science \& Technology B: Microelectronics and Nanometer Structures Processing, Measurement, and Phenomena 19, 2757 (2001); https://doi.org/10.1116/1.1420198

Fabrication of frequency-selective surfaces using microlens projection photolithography Applied Physics Letters 80, 3500 (2002); https://doi.org/10.1063/1.1477941

Infrared mesh filters fabricated by electron-beam lithography Journal of Vacuum Science \& Technology B: Microelectronics Processing and Phenomena 3, 268 (1985); https:// doi.org/10.1116/1.583243

Estimation of scattered particle exposure in ion beam aperture array lithography Journal of Vacuum Science \& Technology B: Microelectronics and Nanometer Structures Processing, Measurement, and Phenomena 24, 2915 (2006); https://doi.org/10.1116/1.2366619

Polarization and angle insensitive dual-band bandpass frequency selective surface using all-dielectric metamaterials

Journal of Applied Physics 119, 134104 (2016); https://doi.org/10.1063/1.4945374

Optical transmission properties of perforated metal films in the middle-infrared range

Applied Physics Letters 94, 081118 (2009); https://doi.org/10.1063/1.3088858 


\title{
Comparison of infrared frequency selective surfaces fabricated by direct-write electron-beam and bilayer nanoimprint lithographies
}

\author{
Irina Puscasu ${ }^{\text {a) }}$ and G. Boreman \\ CREOL/School of Optics, University of Central Florida, Orlando, Florida 32816-2700 \\ R. C. Tiberiob) and D. Spencer \\ Cornell Nanofabrication Facility, Cornell University, Ithaca, New York 14853-5403 \\ R. R. Krchnavek ${ }^{\mathrm{c}}$ \\ Rowan University, Glassboro, New Jersey 08028
}

(Received 1 June 2000; accepted 25 August 2000)

\begin{abstract}
We report on the fabrication of crossed-dipole resonant filters by direct-write electron-beam and nanoimprint lithographies. Such structures have been used as spectrally selective components at visible, microwave, and infrared wavelengths. Imprinting is accomplished in a modified commercial hot press at $155^{\circ} \mathrm{C}$. The replica is then etched in oxygen plasma and developed in chlorobenzene to selectively dissolve the poly(methylmethacrylate and methacrylic acid) and poly(methylmethacrylate) bilayer resist. This step enhances undercut and improves lift-off metalization. Infrared fourier transform spectroscopy was performed to characterize the transmission response of the frequency selective surfaces (FSSs) fabricated. The resonant behavior for the direct-write FSS was found to be $5.3 \mu \mathrm{m}$ and for the nanoimprinted FSS to be $6 \mu \mathrm{m}$. The shift towards longer wavelengths is consistent with the dimensions obtained for the FSSs elements in both cases. (c) 2000 American Vacuum Society. [S0734-211X(00)09206-4]
\end{abstract}

\section{INTRODUCTION}

There are many applications of frequency selective surfaces (FSSs) for microwave communication systems in which the periodic structure helped produce a more efficient reflector antenna. ${ }^{1}$ FSSs are used for the microwave region in radome design. ${ }^{2-4}$ Metal meshes have been used as mirrors for improving the pumping efficiency in optically pumped infrared (IR) lasers. ${ }^{5,6}$ FSSs have also been used in the farinfrared region as beam splitters, ${ }^{7,8}$ filters, ${ }^{9,10}$ polarizers, ${ }^{11,12}$ output couplers for lasers, ${ }^{13}$ and Fabry-Perot interferometers. ${ }^{14,15}$ Continuing advancements in lithography have allowed the element dimensions to be scaled down, thus obtaining FSSs with response in the IR portion of the spectrum. Resonant responses have been demonstrated for arrays of crosses at $7-9,{ }^{16} 6.5,{ }^{17}$ and $1.5 \mu \mathrm{m}^{18}$ and arrays of dipoles at $4-14 \mu \mathrm{m} .^{19}$

Fabrication of resonant arrays for the near-infrared range requires $100 \mathrm{~nm}$ line resolution and lengths and spacings of the elements on the order of micrometers or less. Such resolution can be easily attained by direct-write electron electronbeam lithography (DEBL). Since DEBL is based on exposure of the resist point by point in a serial manner, which gives a low throughput, it is not yet practical for mass production of $100 \mathrm{~nm}$ features. Writing a $1 \mathrm{~cm}$ by $1 \mathrm{~cm}$ cross array by DEBL takes approximately $1 \mathrm{~h}$ running at $25 \mathrm{MHz}$ on a Leica VB6.

Several manufacturing techniques are being considered to meet the demand for fast, reliable, and cost-effective nanolithography. One of the promising alternatives would be

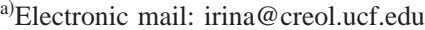

b)Electronic mail: tiberio@cnf.cornell.edu

${ }^{c}$ Electronic mail: krchnavek@ rowan.edu
}

masked ion beam lithography (MIBL). ${ }^{18}$ The parallel processing of MIBL allows for a $1 \mathrm{~cm}^{2}$ area to be written in a single exposure of $20 \mathrm{~s}$ duration. MIBL has higher throughput and is not hindered by the proximity effect, which causes problems with linewidth control in DEBL. One of the disadvantages of the MIBL technique is the fragility of the masks. Also, since the tool has not reached the same advanced stage of development as DEBL, it is not commercially available.

Another possible solution to nanostructure manufacturing is nanoimprint lithography (NIL). ${ }^{20,21}$ This technique is based on the physical deformation of the resist cast on a substrate by a mask with submicron structures on its surface. It offers a cost-effective alternative to DEBL.

In our present investigation of the nanoimprint technique, we have found that a bilayer resist configuration provides the undercut profile that improves the metal lift-off, ${ }^{22}$ enabling one to preserve the resolution obtained after the press. We compare the resonant responses of crossed-dipole arrays fabricated by direct-write e-beam and nanoimprint lithography techniques.

\section{FSSS FABRICATED USING DEBL}

The crossed dipole FSS is fabricated on a $n$-doped $\mathrm{Si}$ wafer of $5 \Omega \mathrm{cm}$ resistivity and $380 \mu \mathrm{m}$ thickness. A singlelayer $495 \mathrm{k}$ MW poly(methylmethacrylate) (PMMA) ${ }^{23}$ resist was spun to a thickness of $150 \mathrm{~nm}$. This was exposed by a focused electron beam using the Leica VB-6 electron-beam pattern generator, at a voltage of $100 \mathrm{kV}$ and a dose of 600 $\mu \mathrm{C} / \mathrm{cm}^{2}$. The resist was developed in a 1:1 solution of methyl isobutyl ketone and isopropanol, for $60 \mathrm{~s}$. A $30 \mathrm{~nm}$ film of gold was then deposited by e-beam evaporation. The metal lift-off was performed by ultrasonic agitation in methylene 


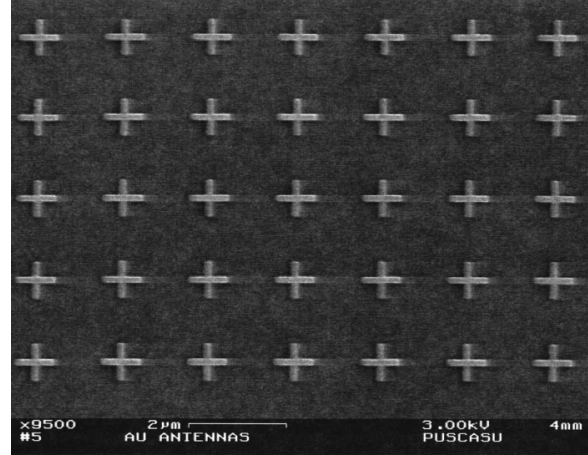

FIG. 1. Scanning electron micrograph of a crossed-dipole array fabricated by DEBL.

chloride. The arm length, $L$, of the crosses, is $850 \mathrm{~nm}$ and the width, $b$, is $140 \mathrm{~nm}$ (Fig. 1). The overall array dimension is $1 \mathrm{~cm}$ by $1 \mathrm{~cm}$.

\section{FSSs FABRICATION USING NIL}

\section{A. Mask fabrication}

The first step in the fabrication of the mask (Fig. 2) for nanoimprint lithography is similar to the fabrication of the actual crossed-dipole FSS by DEBL. The features are written into a single layer of 495k MW PMMA resist on a silicon wafer and the resist is developed in 1:1 solution of methyl isobutyl ketone and isopropanol for 60 s. A $100 \mathrm{~nm}$ chromium-nickel alloy is evaporated over the patterned resist and subsequently lifted-off using methylene chloride. A parallel plate reactive ion etching (RIE) in $\mathrm{CHF}_{3}$ and $\mathrm{CF}_{4}$ is performed in order to transfer the crossed-dipole array pattern into the silicon substrate (Fig. 3). We have found that using equal flow rates, $20 \mathrm{sccm}$, of $\mathrm{CHF}_{3}$ and $\mathrm{CF}_{4}$, for a power of $90 \mathrm{~W}$ and direct current (dc) bias of $585 \mathrm{~V}$, gives nearly vertical walls. Etching was done such that the height of the features obtained was $250 \mathrm{~nm}$.

To improve the release of the replica from the mask usually a thin layer of mold-release compound is deposited on the mask prior to the imprinting. In our work we coated the imprinter by using a low power $\mathrm{CHF}_{3}$ RIE step for $30 \mathrm{~s}$. We

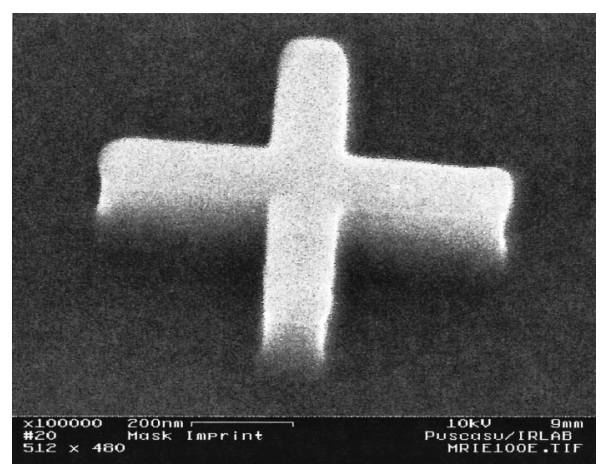

FIG. 2. Crossed-dipole silicon imprint mask.

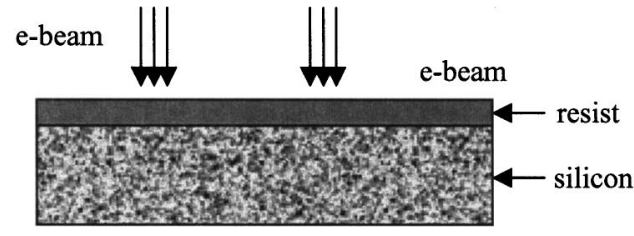

(a)

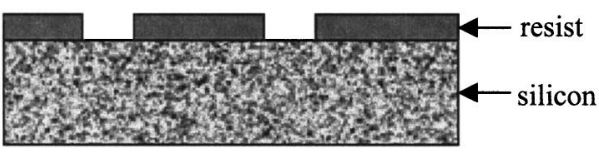

(b)

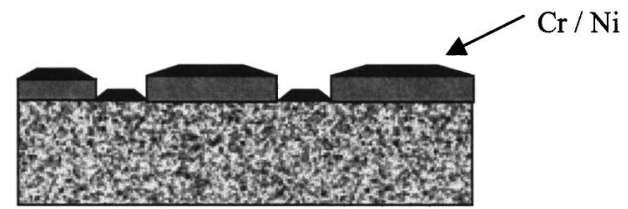

(c)

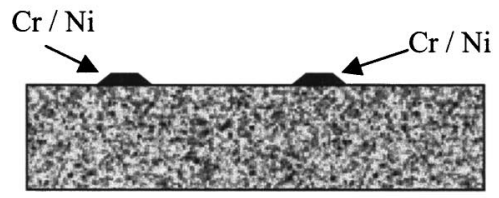

(d)

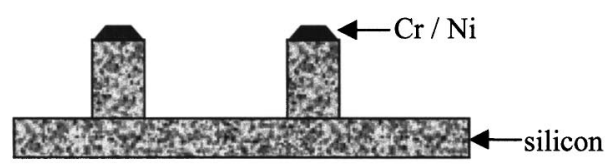

(e)

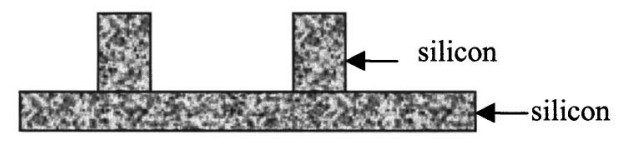

(f)

FIG. 3. Fabrication of the imprint mask (a) exposing the resist, (b) developing the resist, (c) evaporating $\mathrm{Cr} / \mathrm{Ni}$, (d) lift-off, (e) reactive ion etching into the silicon, and (f) $\mathrm{Cr} / \mathrm{Ni}$ etch.

believe this leaves a thin layer of fluorocarbon on the imprinter that protected the mask and avoided permanent sticking to the replica.

\section{B. Imprinting}

NIL is different from both the e-beam and ion-beam lithography in that it does not use energetic beams. The effects of diffraction and scattering of a beam are eliminated. The features are patterned through a mechanical process rather than a chemical change of the resist.

Previous work by Hatzakis $e t$ al. $^{24}$ in e-beam lithography demonstrated that using a bilayer resist can produce an undercut that improves the lift-off process. In a similar fashion, we are using a bilayer configuration to improve the NIL. The replica silicon substrate is identical to the one used for the direct-write e-beam FSS. A layer of 495k MW PMMA 2\% in anisole is spun on the wafer at $2000 \mathrm{rpm}$ for $60 \mathrm{~s}$ and soft 


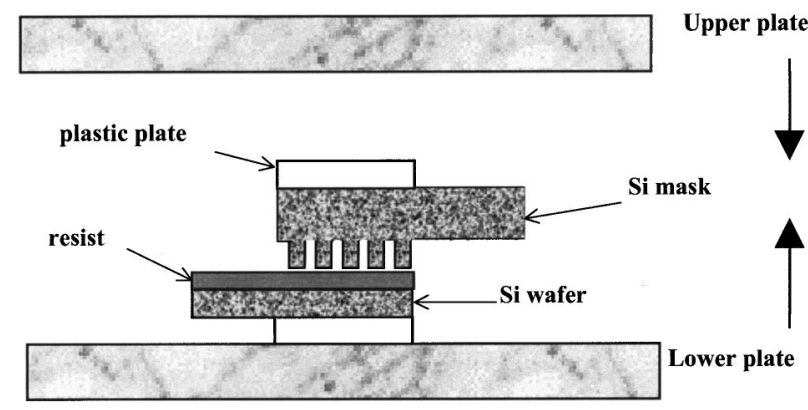

FIG. 4. Nanoimprint press.

baked for $20 \mathrm{~min}$ at $170{ }^{\circ} \mathrm{C}$. This results in a $90 \mathrm{~nm}$ thickness for the first layer resist. The second layer of resist is poly(methylmethacrylate and methacrylic acid) (P(MMAMAA) copolymer) $8.5 \%$ and is spun at $4140 \mathrm{rpm}$ for $60 \mathrm{~s}$, then soft baked for $20 \mathrm{~min}$ at $170{ }^{\circ} \mathrm{C}$, resulting in a $50-\mathrm{nm}$ thick layer. The two types of resist were chosen for their different chemical properties, which prevents their intermixing and will play an important role in the post processing, particularly in the formation of the undercut sidewalls of the features. ${ }^{24}$

To perform the imprint (Fig. 4), we used a generic hot press which uses a compressed air piston. The upper and lower plates of the press have independent temperature control. In order to obtain a uniform pressure over the entire sample area, we used plastic (bulk PMMA) plates to act as cushions between both the silicon mask and the replica wafer and their respective hot plates. At the temperature at which the imprinting is performed, the plastic plates become soft and help insure the mask and the replica are parallel to each other by deforming to compensate for any macroscopic pressure gradients.

The mask and the replica are placed in contact and heated to $100{ }^{\circ} \mathrm{C}$. The mask is then pressed against the resist and the temperature is increased to $155^{\circ} \mathrm{C}$, above the glass transition temperatures of the PMMA and P(MMA-MAA). The optimum force was found to be $560 \mathrm{~N}$ for a $1 \mathrm{~cm}^{2}$ mask. As the silicon crosses of the mask are pressed into the resist, the polymer flows into the adjacent spaces. The mask and the replica are kept under pressure for approximately $10 \mathrm{~min}$ and then flash cooled by dipping them in cold water freezing the pattern into the resist. The thin layer of mold release on the mask helps separate the mask from the replica.

\section{Postprocessing}

After the pattern is imprinted into the bilayer resist on the replica silicon wafer, dark field optical microscopy shows nonuniformity in the depth of the imprinting. The residual resist remaining in the trenches of the imprinting pattern must be removed for lift-off to be successful. We removed the residual resist by using an isotropic etch in an oxygen plasma barrel etcher for approximately $1 \mathrm{~min}$. For the bilayer imprint process, the necessary depth and the undercut is obtained by selectively dissolving the two layers of resist on the replica wafer. Immersing the sample in chlorobenzene,

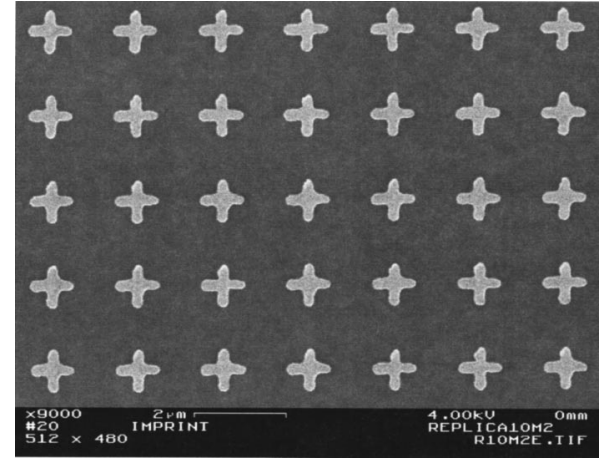

FIG. 5. Scanning electron micrograph of a crossed-dipole array fabricated by NIL.

for $60 \mathrm{~s}$ selectively and isotropically dissolves approximately $60 \mathrm{~nm}$ of the bottom layer of PMMA. Since the P(MMAMAA) is not soluble in chlorobenzene, it would remain intact. ${ }^{22}$ Once the undercut is obtained, a thin layer of gold is evaporated onto the replica, followed by the lift-off to obtain the FSS (Fig. 5).

Inspection shows a process bias of $+30 \mathrm{~nm}$ of the replica with respect to the imprinter. We believe this is due to the isotropic etch in the oxygen plasma barrel etcher. This step, and the chlorobenzene soak improved our yield. The use of a commercial press and postprocessing ensured high yield and cost-effective imprinting. Improvements in the stability of the imprinting machine and in the postprocessing, for example using an anisotropic etch, could decrease the bias.

\section{SPECTRAL MEASUREMENTS}

The spectral transmission of the FSSs fabricated by direct or imprint was measured at normal incidence over the 3-12 $\mu \mathrm{m}$ band using a Perkin-Elmer 1710 infrared fourier transform spectrometer, at a spectral resolution of $4 \mathrm{~cm}^{-1}$. Discussion of the measurement issues have been presented previously. ${ }^{19}$

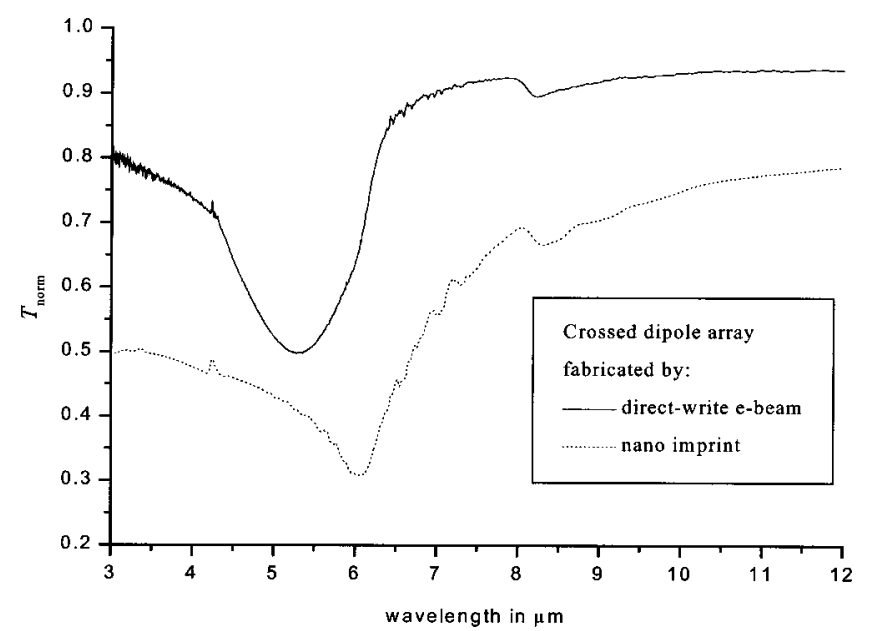

FIG. 6. Normalized transmittance for direct-write and nanoimprint crossdipole arrays. 
TABLE I. Theoretical and experimental values of the resonant wavelength of the direct-write and nanoimprint FSS, respectively.

\begin{tabular}{lcccc}
\hline \hline & & & $\lambda_{\text {res }}=2.1 L\left(1+\frac{b}{2 L}\right) n_{\text {eff }}$ & $\lambda_{\text {res }}$ \\
\multicolumn{1}{c}{ FSS } & $L(\mu \mathrm{m})$ & $b(\mu \mathrm{m})$ & $(\mu \mathrm{m})$ & $(\mu \mathrm{m})$ \\
\hline Direct-write & 0.85 & 0.14 & 4.87 & 5.3 \\
Imprint & 0.94 & 0.2 & 5.5 & 6 \\
\hline \hline
\end{tabular}

We emphasize the resonant nature of the spectral features by plotting the spectral transmittance (Fig. 6) of each FSS normalized to that of the substrate

$$
T_{\text {norm }}=\frac{T_{\text {FSS }}}{T_{\text {substrate }}} .
$$

For a single narrow strip, the wavelength of resonance in transmission is given approximately by ${ }^{25}$

$$
\lambda_{0, \mathrm{res}}=2.1 L\left(1+\frac{b}{2 L}\right),
$$

where $L$ and $b$ represent the length and the width, respectively, of the strip.

For a configuration of substrates with a refractive index $n_{1}$ above and $n_{2}$ below, the actual wavelength of resonance is modified approximately by the effective index $n_{\text {eff }}$ of the two-layer medium

$$
\lambda_{\text {res }}=\lambda_{0, \text { res }} n_{\text {eff }}
$$

with $n_{\text {eff }}$ is given by

$$
n_{\mathrm{eff}}=\left(\frac{n_{1}^{2}+n_{2}^{2}}{2}\right)^{1 / 2} \text {. }
$$

In our case, the medium above is air and the medium below is the silicon wafer. Using an approximate index of 3.42 for the silicon at the particular resonant wavelengths, we obtain the values for the wavelength of resonance in Table I. The resonant response of the imprinted array is slightly shifted towards longer wavelengths due to an increase in the length of the imprinted feature compared to the direct-write e-beam FSS or the imprinted mask. Despite the wider features obtained by imprint, the resonance remains reasonably sharp. We observe also a difference in the transmission level between the two curves, which is due to residual metal remained in small areas over the imprinted sample.

\section{CONCLUSIONS}

We have discussed the fabrication of crossed-dipole resonant filters by direct-write e-beam and nanoimprint lithographies. Nanoimprinting on a $1 \mathrm{~cm}^{2}$ wafer scale has been shown for $200 \mathrm{~nm}$ width crossed dipoles. Imprinting was accomplished in a modified commercial hot press at $155^{\circ} \mathrm{C}$.
The imprinted structures have been processed with oxygen plasma etching and chlorobenzene to remove the remaining resist from the trenches. A bilayer resist has been used to obtain the undercut sidewalls needed for the lift-off to have high yield. The optical performance of the FSSs fabricated was measured. The resonant behavior was found at $5.3 \mu \mathrm{m}$ for the direct-write sample and at $6 \mu \mathrm{m}$ for the nanoimprint sample. The shift in wavelength is consistent with the dimensions obtained for the array elements in both cases.

Along with masked ion beam lithography, nanoimprinting will offer a fast and cost effective alternative to DEBL in fabrication of IR filters and with further development, could become a commercial technology for manufacturing nanostructures.

\section{ACKNOWLEDGMENTS}

This work was performed in part at the Cornell Nanofabrication Facility (a member of the National Nanofabrication Users Network) which is supported by the National Science Foundation under Grant No. ECS-9731293, Cornell University, and industrial affiliates.

${ }^{1}$ F. O'Nians and J. Matson, US Patent No. 3, 231, 892 (Jan. 1966).

${ }^{2}$ S. W. Lee, IEEE Trans. Antennas Propag. AP-19, 656 (1971).

${ }^{3}$ B. A. Munk et al., IEEE Trans. Antennas Propag. AP-22, 804 (1974).

${ }^{4}$ R. Pous and D. M. Pozar, Electron. Lett. 25, 1136 (1989).

${ }^{5}$ E. J. Danielewicz, T. K. Plant, and T. A. DeTemple, Opt. Commun. 13, 366 (1975).

${ }^{6}$ M. S. Durschlag and T. A. DeTemple, Appl. Opt. 20, 1245 (1981).

${ }^{7}$ P. Vogel and L. Genzel, Infrared Phys. 4, 257 (1964).

${ }^{8}$ P. A. R. Ade, A. E. Costley, C. T. Cunningham, C. L. Mok, G. L. Neill, and T. J. Parker, Infrared Phys. 19, 599 (1979).

${ }^{9}$ A. Mitsuishi, Y. Otsuka, S. Fujita, and H. Yoshinaga, Jpn. J. Appl. Phys., Part 1 9, 574 (1963).

${ }^{10}$ G. D. Holah and N. Morrison, J. Opt. Soc. Am. 67, 971 (1977).

${ }^{11}$ A. E. Costley, K. H. Hursey, G. F. Neill, and J. W. M. Ward, J. Opt. Soc. Am. 67, 979 (1977).

${ }^{12}$ C. L. Mok, W. G. Champers, T. J. Parker, and A. E. Costley, Infrared Phys. 19, 437 (1979).

${ }^{13}$ R. Ulrich, T. J. Bridges, and M. A. Pollack, Appl. Opt. 9, 2511 (1970).

${ }^{14}$ R. Ulrich, K. F. Renk, and L. Genzel, IEEE Trans. Microwave Theory Tech. MTT-11, 363 (1963).

${ }^{15}$ V. Ya. Balakhanov, Sov. Phys. Tech. Phys. 10, 788 (1966).

${ }^{16}$ C. M. Rhoades, E. K. Damon, and B. A. Munk, Appl. Opt. 21, 2814 (1982).

${ }^{17}$ D. M. Byrne, A. J. Brouns, F. C. Case, R. C. Tiberio, B. L. Whitehead, and E. D. Wolf, J. Vac. Sci. Technol. B 3, 268 (1985).

${ }^{18}$ M. D. Morgan, W. E. Horne, V. Sundaram, J. C. Wolfe, S. V. Pendharkar, and R. Tiberio, J. Vac. Sci. Technol. B 14, 3903 (1996).

${ }^{19}$ I. Puscasu, D. Spencer, and G. Boreman, Appl. Opt. 39, 1561 (2000).

${ }^{20}$ J. L. Wilbur, A. Kumar, E. Kim, and G. M. Whitesides, Adv. Mater. 6, 600 (1994).

${ }^{21}$ S. Y. Chou, P. R. Krauss, and P. J. Renstrom, Appl. Phys. Lett. 67, 3114 (1995).

${ }^{22}$ B. Faircloth, H. Rohrs, R. Tiberio, R. Ruoff, and R. R. Krchnavek, J. Vac. Sci. Technol. B 18, 1866 (2000).

${ }^{23}$ Micro-Chemical Corporation, Newton, MA.

${ }^{24}$ M. Hatzakis, D. Hofer, and T. H. P. Chang, J. Vac. Sci. Technol. 16, 1631 (1979).

${ }^{25}$ S. T. Chase and R. D. Joseph, Appl. Opt. 22, 1775 (1983). 